\title{
Hydrodynamic Stabilization of Self-Organized Criticality in a Driven Rydberg Gas
}

\author{
K. Klocke $\odot,{ }^{1,2}$ T. M. Wintermantel $\odot,{ }^{3,4}$ G. Lochead, ${ }^{3}$ S. Whitlock $\odot,{ }^{3}$ and M. Buchhold $\oplus^{1,5}$ \\ ${ }^{1}$ Department of Physics and Institute for Quantum Information and Matter, California Institute of Technology, \\ Pasadena, California 91125, USA \\ ${ }^{2}$ Department of Physics, University of California, Berkeley, California 94720, USA \\ ${ }^{3}$ ISIS (UMR 7006), University of Strasbourg and CNRS, 67000 Strasbourg, France \\ ${ }^{4}$ Physikalisches Institut, Universität Heidelberg, 69120 Heidelberg, Germany \\ ${ }^{5}$ Institut für Theoretische Physik, Universität zu Köln, D-50937 Cologne, Germany
}

(Received 29 September 2020; accepted 18 February 2021; published 23 March 2021)

\begin{abstract}
Signatures of self-organized criticality (SOC) have recently been observed in an ultracold atomic gas under continuous laser excitation to strongly interacting Rydberg states [S. Helmrich et al., Nature, 577, 481-486 (2020)]. This creates unique possibilities to study this intriguing dynamical phenomenon under controlled experimental conditions. Here we theoretically and experimentally examine the self-organizing dynamics of a driven ultracold gas and identify an unanticipated feedback mechanism originating from the interaction of the system with a thermal reservoir. Transport of particles from the flanks of the cloud toward the center compensates avalanche-induced atom loss. This mechanism sustains an extended critical region in the trap center for timescales much longer than the initial self-organization dynamics. The characteristic flattop density profile provides an additional experimental signature for SOC while simultaneously enabling studies of SOC under almost homogeneous conditions. We present a hydrodynamic description for the reorganization of the atom density, which very accurately describes the experimentally observed features on intermediate and long timescales, and which is applicable to both collisional hydrodynamic and chaotic ballistic regimes.
\end{abstract}

DOI: 10.1103/PhysRevLett.126.123401

Introduction.-Many-body systems, may they be driven, open, or excited by a sudden parameter quench, often evolve toward steady or transient metastable states which can be classified as far from thermal equilibrium [1-13]. Sometimes these systems feature attractors for the nonequilibrium dynamics that give rise to emergent scale invariant properties over a wide range of initial states or parameters [14-18]. One paradigmatic example is selforganized criticality (SOC), whereby a dissipative manybody system evolves toward a (nonequilibrium) critical state by an intrinsic feedback mechanism. Since its first introduction by Bak, Tang, and Wiesenfeld in 1987 [12,19], SOC has been intensively studied theoretically and associated with phenomena ranging from avalanches and earthquakes to solar flares and neuronal activity [20-24].

The range of phenomena found to exhibit SOC-like characteristics is at odds however with the relatively stringent conditions expected to lead to SOC [25]. For example, the typical requirements of a large separation of timescales between slow dissipation and fast, conservative bulk dynamics will never be perfectly satisfied in practice [26]. This has lead to the notion of self-organized quasicriticality (SOQC) where the system hovers around criticality with large excursions into the sub- and supercritical phases [26,27]. Nonetheless, key signatures of SOC including scale invariance of the stationary density and power-law distributed excitation avalanches were recently observed in the driven-dissipative dynamics of atomic Rydberg gases [28] (see also related experiments in driven thermal gases [29]). These works, however, did not pinpoint an integral mechanism which brings the system out of the subcritical absorbing phase. This therefore raises important questions about how signatures of the SOC state persist for long times and whether it bears universal characteristics [25] that can be extracted from experiments in a transient regime.

Here we experimentally and theoretically demonstrate that the mechanisms leading to the SOC state are remarkably robust. We show that slow thermal motion of the particles provides an additional feedback mechanism which stabilizes the system close to the critical state over an extensive time period. This is evidenced by the experimental observation of a stable flattop profile in the atomic gas, where the wings of the distribution act as particle reservoirs that compensate particle loss in the trap center (Fig. 1). To explain this result we develop a hydrodynamic Langevin equation which includes the competition between thermalization of the gas (in the motional degrees of freedom) and the driven-dissipative excitation dynamics leading to SOC. The thermal motion of each atom in the trapping potential yields an effective evolution for the atom density, which we describe in the overdamped regime, 


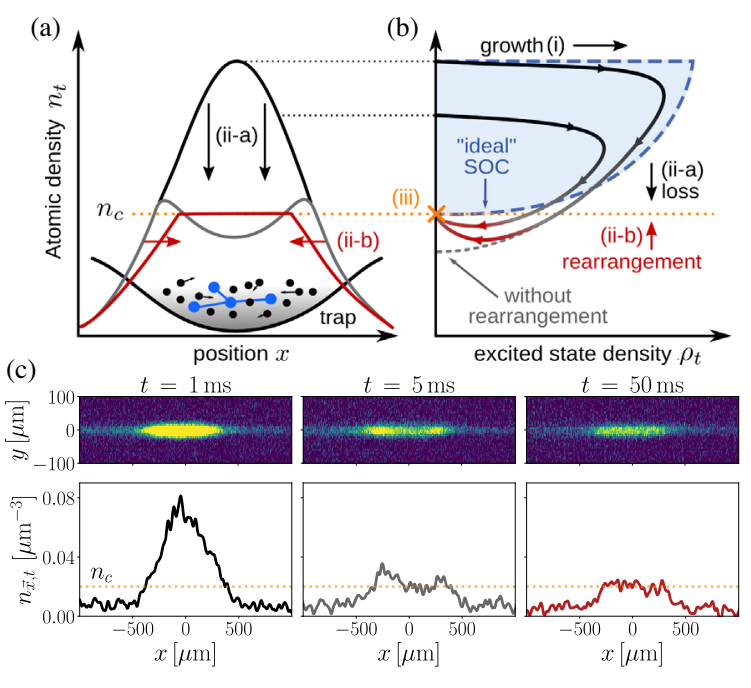

FIG. 1. Mechanisms for self-organized criticality in an ultracold atomic gas. (a) A trapped atomic gas with inhomogeneous density distribution is continuously driven by an off-resonant excitation laser to highly excited Rydberg states (blue disks). (b) Trajectory of the atom density $n_{t}$ and the excitation density $\rho_{t}$ driven by facilitated excitation, decay, and hydrodynamic motion. Starting from the supercritical phase $n_{t=0}>n_{c}$ the system undergoes: (i) rapid growth of Rydberg density; (ii-a) selforganization from the active phase toward the critical point via gradual depletion of particles (caused by loss from the Rydberg state); (ii-b) refilling of the central density from the subcritical phase by atomic rearrangement (thermal motion) from the wings to the trap center; (iii) stabilization close to the critical point for an extended period of time. (c) Upper panels: exemplary experimental absorption images $\left(n_{\vec{x}, t}\right.$ integrated over $z$ ) at different times. Lower panels: Reconstructed three-dimensional atom density $n_{\vec{x}, t}$ at $y=z=0$ showing a flattop profile at the SOC critical density starting around $5 \mathrm{~ms}$ and persisting until much later times.

giving rise to drift and diffusion terms. Although the experiment is not in a collisional regime, this approach captures the collective evolution towards a thermal distribution extremely well. It is reminiscent of the hydrodynamics of a gas in classical statistical mechanics and gives rise to density currents. This allows the cloud to adapt by slowly refilling subcritical regions back to a critical state, similar to plasticity in biological neural networks [30,31].

Self-organization mechanism.-We consider a spatially inhomogeneous gas of ultracold atoms held in an approximately harmonic optical potential produced by a focused far-off-resonant laser beam [28] [depicted in Fig. 1(a)]. The atoms are continuously driven by a detuned laser field, which creates rare and isolated Rydberg excitations at random positions in the gas. Once an excitation is present it either spontaneously decays (typically accompanied by loss from the trap [28]), or triggers secondary excitations through a process called Rydberg facilitation [32-35]. This occurs at a characteristic distance $r_{\text {fac }} \approx 4.5 \mu \mathrm{m}$ (for the present experiments) where the laser detuning is compensated by the van der Waals interaction between Rydberg pair states [36]. The self-organizing dynamics are driven by the competition between facilitated excitation (with a rate proportional to $\kappa n_{\vec{x}, t}$, where $\kappa$ is the microscopic facilitation rate per unit volume and $n_{\vec{x}, t}$ is the total atom density) and decay of excitations with a density independent, overall decay rate per excited atom $\Gamma$. These two processes compete to produce complex dynamics [28,37-40] which drives the system to a critical atom density $n_{c} \approx \Gamma / \kappa$. For $n_{\vec{x}, t}>n_{c}$ (supercritical or active phase) individual excitations can grow into spatially extended clusters of excitations (avalanches) with a high degree of activity and particle loss. For $n_{\vec{x}, t}<n_{c}$ (absorbing phase), these excitation avalanches are rare or vanishingly small.

Figure 1 illustrates the mechanisms leading to SOC. Starting from the supercritical regions of the cloud, the density of excitations $\rho_{\vec{x}, t}$ undergoes a period of rapid growth [labeled (i) in Fig. 1(b)] $\left(\kappa n_{\vec{x}, t}>\Gamma\right)$. Roughly after one millisecond $(\approx 1 / \Gamma)$, this is followed by a slow, visible decrease in both $n_{\vec{x}, t}$ and $\rho_{\vec{x}, t}$ owing to a gradual loss of excited atoms [labeled (ii-a)]. In the limit of vanishingly small effective loss rate (perfect separation of timescales) the system will follow a characteristic trajectory [dashed blue curve in Fig. 1(b)] that terminates at the critical point [orange cross at $n_{\vec{x}, t}=n_{c}$ and $\rho_{\vec{x}, t}=0$ ]. If instead the excitation avalanches persist on timescales comparable to the time for self-organization, the dynamics may overshoot the critical point, terminating in the absorbing phase [dotted grey curve in Fig. 1(b)]. This is associated with the appearance of a temporary dip in the atomic density distribution [grey curve in Fig. 1(a)]. However, slow motion of particles in the trap refills this density dip, providing a mechanism to escape the absorbing phase and approach the critical point [red curve in Fig. 1(a), labeled (ii-b)]. This interplay of nonlinear excitation dynamics and atomic motion explains how the system self-organizes close to the critical point with a constant critical density across the cloud and sustains critical dynamics (e.g., avalanches) for long times compared to the initial selforganization period [labeled (iii)].

Experimental approach.-Our experiments start with an ultracold gas of $N=10^{5}$ potassium-39 atoms trapped in a cigar-shaped optical potential with trap frequencies $\omega_{x} / 2 \pi=65 \mathrm{~Hz}$ and $\omega_{y, z} / 2 \pi=950 \mathrm{~Hz}$. The atomic cloud has a temperature $T=40 \mu \mathrm{K}$ and $e^{-1 / 2}$ radii $\sigma_{x}=210 \mu \mathrm{m}$, $\sigma_{y, z}=12 \mu \mathrm{m}$ with a peak density $n_{0}=0.21 \mu \mathrm{m}^{-3}$. At time $t=0$, we switch on an off-resonant ultraviolet (UV) laser coupling with Rabi frequency $\Omega / 2 \pi \approx 200 \mathrm{kHz}$ and detuning $\Delta / 2 \pi=30 \mathrm{MHz}$ on the transition from the ground state $|g\rangle=\left|4 s_{1 / 2}, F=1\right\rangle$ to the Rydberg state $|r\rangle=\left|66 p_{3 / 2}\right\rangle$. To strongly suppress single-particle excitations and ensure that many-body effects dominate, we stay in the regime $\Gamma \ll \Omega \ll \Delta[37,41-52]$. Excitations decay with a calculated rate $\Gamma / 2 \pi=0.84 \mathrm{kHz}$, which 
either brings them back to the ground state $|g\rangle$ or into states $|0\rangle$ which are decoupled or lost from the trap. This irreversible loss of particles $|r\rangle \rightarrow|0\rangle$ provides the first crucial self-organization mechanism [28]. After laser exposure time $t$, we measure the number of Rydberg excitations in the cloud as well as the spatial distribution of ground state atoms remaining in the trap. For the former we field ionize the Rydberg atoms and detect them on a microchannel plate detector. For the latter we take an absorption image of the atom cloud, which integrates along the propagation of the light field [28].

Example absorption images after different exposure times $t$ are shown at the top of Fig. 1(c), roughly coinciding with those sketched in Fig. 1(a). The line profiles in Fig. 1(c) are reconstructed cross sections of the threedimensional density distribution through the center of the cloud. They are obtained by an inverse Abel transformation of the average over several absorption images. Initially the gas has an approximately Gaussian shape as expected for an optically trapped thermal gas. At $t=5 \mathrm{~ms}$, a dip in the center where the density was initially the highest has developed. For even longer times $t \gtrsim 15 \mathrm{~ms}$, this dip has filled in to give a flattop coinciding with the critical density $n_{c}$.

Theoretical description.-The collective dynamics of the driven Rydberg ensemble can be described by a nonequilibrium field theory for the local density of particles $n_{\vec{x}, t}$ and the density of excitations $\rho_{\vec{x}, t}$ [28]. Besides dissipative decay and facilitated spreading of excitations, we also account for hydrodynamic motion of the atoms via two coupled stochastic evolution equations for $\rho_{\vec{x}, t}, n_{\vec{x}, t}$, including the internal and external degrees of freedom.

We label each atom with an index $j$, a set of operators $\sigma_{j}^{\alpha \beta}=|\alpha\rangle\left\langle\left.\beta\right|_{j}\right.$ where $\alpha, \beta$ label the states $0, g, r$, and a (classical) position $\vec{x}_{l}$. The equation of motion (EOM) for the internal degrees of freedom is given by the microscopic Liouvillian

$$
\begin{aligned}
\partial_{t} \sigma_{l}^{\alpha \beta}= & i\left[\left(\sum_{j \neq l} \frac{C_{6} \sigma_{j}^{r r}}{\left|\vec{x}_{l}-\vec{x}_{j}\right|^{6}}-\Delta\right) \sigma_{l}^{r r}+\Omega \frac{\sigma_{l}^{r g}+\sigma_{l}^{g r}}{2}, \sigma_{l}^{\alpha \beta}\right] \\
& +\delta_{\alpha \beta}\left(\delta_{\alpha r} \gamma_{\mathrm{de}}+\delta_{\alpha g} \gamma_{\downarrow g}+\delta_{\alpha 0} \gamma_{\downarrow 0}\right) \sigma_{l}^{\alpha \alpha}-\frac{\Gamma}{2}\left\{\sigma_{l}^{r r}, \sigma_{l}^{\alpha \beta}\right\},
\end{aligned}
$$

with the anti-commutator $\{\cdots, \cdots\}$, commutator $[\cdots, \cdots]$, and Kronecker symbol $\delta_{\alpha, \beta}$. This includes coherent singleparticle processes: laser driving with Rabi frequency $\Omega$ and detuning $\Delta$, and an (effectively) isotropic van der Waals interaction between atoms $l$ and $j$ if both are in the Rydberg state (assuming an average over Rydberg pair state potentials [53]). Dissipative single-particle processes are quantified by the dephasing rate $\gamma_{\mathrm{de}}$, the spontaneous decay rate $\gamma_{\downarrow g}$ for the process $|r\rangle \rightarrow|g\rangle\left(\gamma_{\downarrow 0}\right.$ for $\left.|r\rangle \rightarrow|0\rangle\right)$ and $\Gamma=\gamma_{\mathrm{de}}+\gamma_{\downarrow g}+\gamma_{\downarrow 0}$, where $\Gamma \gg \gamma_{\downarrow, 0}$ [28].

Defining a spherical unit cell with radius $r_{\text {fac }}$ and volume $V_{\text {fac }}$, the coarse-grained densities are [28] $\rho_{\vec{x}, t}=\sum_{j, \vec{x}}^{\prime}\left\langle\sigma_{j}^{r r}\right\rangle / V_{\mathrm{fac}}, n_{\vec{x}, t}=\sum_{j, \vec{x}}^{\prime}\left\langle\sigma_{j}^{r r}+\sigma_{j}^{g g}\right\rangle / V_{\mathrm{fac}}$ where $\sum_{j, \vec{x}}^{\prime}$ is restricted to $j$ with $\left|\vec{x}_{j}-\vec{x}\right| \leq r_{\text {fac }}$. The EOM for the atomic density is evaluated by applying the chain rule

$\partial_{t} n_{\vec{x}, t}=\sum_{j, \vec{x}}^{\prime} \frac{\partial_{t}\left\langle\sigma_{j}^{r r}+\sigma_{j}^{g g}\right\rangle}{V_{\mathrm{fac}}}-\nabla \sum_{j, \vec{x}}^{\prime} \frac{\left\langle\sigma_{j}^{r r}+\sigma_{j}^{g g}\right\rangle}{V_{\mathrm{fac}}} \partial_{t} \vec{x}_{j}$,

where $\nabla=\left(\partial_{x}, \partial_{y}, \partial_{z}\right)$. It contains the EOM for the internal degrees of freedom and for the position of the atoms. The sum over the velocities in Eq. (1) is by definition the coarse grained current $\vec{j}$.

An equivalent computation for $\partial_{t} \rho_{\vec{x}, t}$ yields the Langevin equation $[40,54]$

$\partial_{t} \rho_{\vec{x}, t}=\left(D \nabla^{2}-\Gamma\right) \rho_{\vec{x}, t}+\left(\tau+\kappa \rho_{\vec{x}, t}\right)\left(n_{\vec{x}, t}-2 \rho_{\vec{x}, t}\right)+\xi_{\vec{x}, t}$.

Evolution within each unit cell consists of facilitated (de-) excitation with an estimated rate $\kappa \rho_{\vec{x}, t} \approx\left(\Omega^{2} V_{\text {fac }} / 2 \Delta\right) \rho_{\vec{x}, t}$ $[28,54]$ and dissipative decay $\sim \Gamma$. Excitations spread diffusively between unit cells with $D r_{\text {fac }} \approx \kappa$. Rare, off-resonant single-particle excitations occur with rate $\tau r_{\text {fac }}^{3}=(\kappa \Gamma / \Delta) \approx 10^{-4} \kappa$, acting as local seeds to prevent the system from getting stuck in an absorbing state. Local fluctuations in the excitation density are described by a multiplicative Markovian noise $\xi(\vec{x}, t)$ with autocorrelation function $\left\langle\xi(\vec{x}, t) \xi\left(\vec{y}, t^{\prime}\right)\right\rangle=\delta(\vec{x}-\vec{y}) \delta\left(t-t^{\prime}\right)\left(\Gamma \rho_{\vec{x}, t}+\tau\right)$ [54].

The EOM of the density $n_{\vec{x}, t}$ yields

$$
\partial_{t} n_{\vec{x}, t}=-\nabla \vec{j}_{\vec{x}, t}-\gamma_{\downarrow 0} \rho_{\vec{x}, t},
$$

where we use the current $\vec{j}_{\vec{x}, t}=-\left(D_{T} \nabla+\eta \nabla V_{\vec{x}}\right) n_{\vec{x}, t}$, which is obtained from a hydrodynamic (coarse-grained) description of a classical thermal gas [55]. The evolution is composed of diffusion $\left(\propto D_{T}\right)$ and drift due to the external force $-\nabla V_{\vec{x}}$. The force originates from the harmonic trapping potential $V_{\vec{x}}=(M / 2) \sum_{l=x, y, z}\left(\omega_{l} \vec{x}_{l}\right)^{2}$, for which we use the frequencies $\omega_{l}$ and atomic mass $M$ from the experiment. This statistical treatment of the atom motion is motivated by (i) the short thermal deBroglie wavelength $\lambda_{\text {th }}=h / \sqrt{2 \pi M k_{B} T} \approx 30 \mathrm{~nm}$ compared to the trap size $(10-100 \mu \mathrm{m})$ and (ii) an effectively collisionless motion due to the low density and small scattering cross section for the gas.

In the limit $\gamma_{\downarrow 0} \rightarrow 0$, the steady state has zero current $\vec{j}_{\vec{x}, t}=0$ and follows the Gaussian equilibrium distribution $n_{\vec{x}, t}=n_{\vec{x}}^{(\mathrm{eq})}=n_{0} \exp \left(-V_{\vec{x}} / k_{B} T\right)$. For the numerical simulation we use the temperature $T$ measured in the experiment, such that the initial spatial extension of the cloud is $\sigma_{l}=\sqrt{k_{B} T / M \omega_{l}^{2}}$, i.e., $\sigma_{y, z}=2.5 r_{\text {fac }}$ and $\sigma_{x}=44 r_{\text {fac }}$. For $\gamma_{\downarrow 0}>0$ the current counteracts the loss and pulls density towards the center of the cloud, trying to rethermalize to a Gaussian distribution with a typical rate $\eta M \omega_{x}^{2}$. In the EOM 
for $\rho_{\vec{x}, t}$, particle motion is negligible compared to the facilitated spreading of excitations, i.e., $\sim D r_{\mathrm{fac}}^{-2} \gg \eta M \omega_{x}^{2}$.

The mobility $\eta$ is related to the diffusion constant $D_{T}=\eta k_{B} T$ via the Einstein relation and the product $\eta M$ corresponds to the timescale over which the gas thermalizes in the absence of the driving laser. Because the gas is in the collisionless regime, the effective value of $\eta$ is determined primarily by the anharmonicity of the trapping potential. A comparison with the experimental thermalization time yields $\sim 1 /\left(\eta M \omega_{x}^{2}\right)=7 \mathrm{~ms}$. This respects the separation of timescales between the fast spreading of excitations $\sim r_{\text {fac }}^{2} / D=0.1 \mathrm{~ms}$ and a thermalization time of $\approx 2$ (29) oscillation periods in the $x(y-z)$ direction.

The Langevin equations are integrated numerically on a $3+1$-dimensional lattice via an operator splitting scheme $[55,58,59]$. In the simulations we use parameters matching the experimentally observed facilitation and decay rates $[28,60]$.

Dynamics.-Figure 2 shows comparable experiments and numerical simulations for an initially Gaussian atomic cloud with peak density $n>n_{c}$. Examining both components $n_{\vec{x}, t}, \rho_{\vec{x}, t}$ provides insights into the different dynamical regimes. An initial growth regime (i), covering the first few milliseconds of evolution, results in a macroscopic

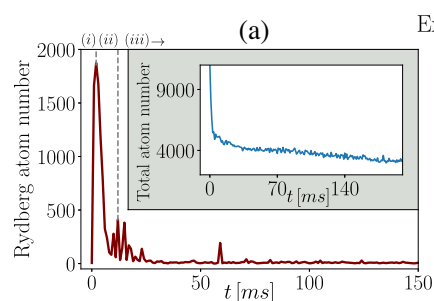

(c)
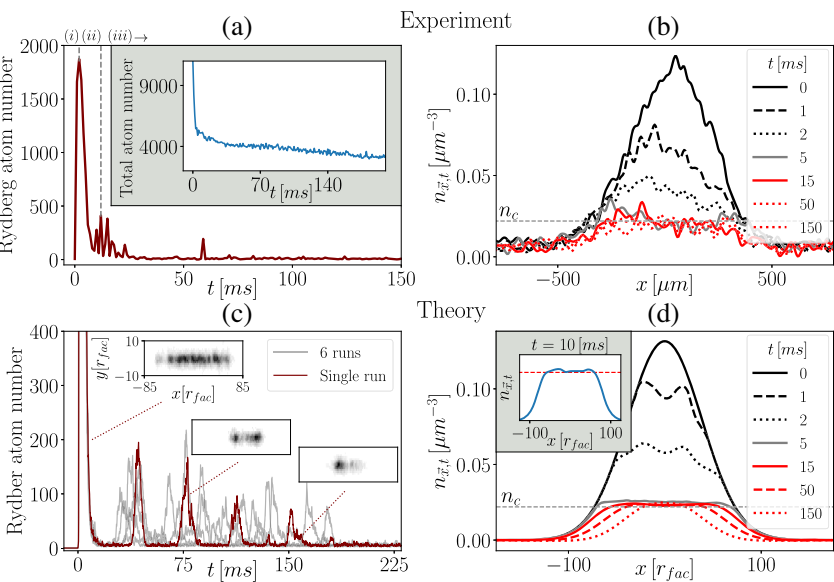

Theory

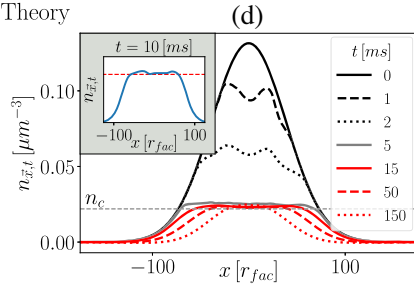

FIG. 2. Theory-experiment comparison showing the approach to the SOC state (top: experiment, bottom: theory). (a) Instantaneous number of Rydberg excitations integrated over the cloud $\left(\propto \rho_{t}\right)$. Each data point is obtained from a destructive measurement and corresponds to a distinct experimental realization. Several small but statistically significant avalanches are observed for $t>50 \mathrm{~ms}$. Inset: corresponding total atom number. (b) Onedimensional slices through the atomic density distribution $n_{\vec{x}, t}$ at $y=z=0$. (c) Simulated dynamics of the full time evolution (red line: single trajectory, grey lines: overlapped data of six different trajectories) showing temporally well separated, extensive excitation avalanches that persist long after the initial growth and self-organizing regimes (i) and (ii). (insets: snapshots of the peak excitation density per avalanche at $z=0$ ). (d) Slices through the simulated density profiles at $y=z=0$ showing the formation of a flattop density profile pinned at $n_{\vec{x}, t}=n_{c}$ analogous to the experimental observations in (b) (see also inset).
Rydberg population. The early time growth dynamics are interesting in their own right [61], but are not overly important for the self-organizing behavior on longer timescales. Subsequent loss from the Rydberg state begins to decrease the total atom number, leading to a self-organizing regime (ii), evidenced by large bursts of Rydberg excitations [large activity seen in Figs. 2(a) and 2(c)] and a sudden drop in the central density of the atomic cloud. The density approaches a flattop distribution with critical central density $n_{\vec{x}, t} \approx n_{c}$. This marks the onset of the selforganized critical regime (iii), characterized by a nearly flat central density $n_{\vec{x}, t}=n_{c}$ [red curves in Figs. 2(b) and 2(d), imaging noise is enhanced by the inverse Abel transform causing the appearance of additional structure in Fig. 2(b)] and sporadic avalanchelike excitation events. This is reached after approximately $15 \mathrm{~ms}$ in the experiment and persists until at least $150 \mathrm{~ms}$. Simulations show that subsequent avalanches are well separated in space and time, implying that experimentally observed Rydberg excitation spikes correspond to individual avalanche events [Fig. 2(c)].

We identify the state reached under these experimental conditions as SOC because it self-organizes to a critical density profile $n_{\vec{x}, t}=n_{c}$. Close to the critical density, the system is known to yield power-law statistics for excitation outbursts [7,59], demonstrated for the same setup in Ref. [28]. In this regime each avalanche transiently imprints a slight depression in the density profile such that $n_{\vec{x}, t}<n_{c}$. However, particle transport from the flanks reestablishes $n_{\vec{x}, t} \approx n_{c}$ between successive avalanches (Fig. 2), thus sustaining a close to ideal critical SOC state over a large region of the system.

To quantify the characteristic timescale for this mechanism, we investigate the effective refilling rate of the central region $\lambda$. A necessary condition for maintaining a SOC state is to satisfy a common separation of timescales $\gamma_{\downarrow 0} \gg \lambda \gg \tau$ [23,59]. The refilling rate is determined by the gradient of the particle current $\lambda n_{\vec{x}, t} \equiv-\nabla \vec{j}_{\vec{x}, t}$ from the wings towards the center. To estimate $\lambda$, we apply a meanfield approach based on our observation that the current is dominated by particle flow along the elongated $x$ direction. Therefore, we assume a quasi-one-dimensional cloud with a flattop of length $L_{x}$ and a constant central density $n_{x, t}=\bar{n}_{t} \geq n_{c}$. Outside, the density is subcritical and follows the equilibrium profile $n_{x, t}=\bar{n}_{t}\left(n_{x}^{(\mathrm{eq})} / n_{L_{x} / 2}^{(\mathrm{eq})}\right)$, which minimizes the current $\vec{j}_{x, t}=0$ in the absence of excitations $\left(\rho_{x, t}=0\right)$. Averaging the current induced particle gain over the center yields

$$
\bar{n}_{t} \lambda=-\frac{1}{L_{x}} \int_{|x| \leq L_{x} / 2} d x \partial_{x} j_{x, t}=\eta M \omega_{x}^{2} \bar{n}_{t} .
$$

Using Eq. (4), we estimate $\gamma_{\downarrow 0} / \lambda \approx 50$ and $\lambda / \tau \approx 100$ from experimental parameters (and comparable for the 

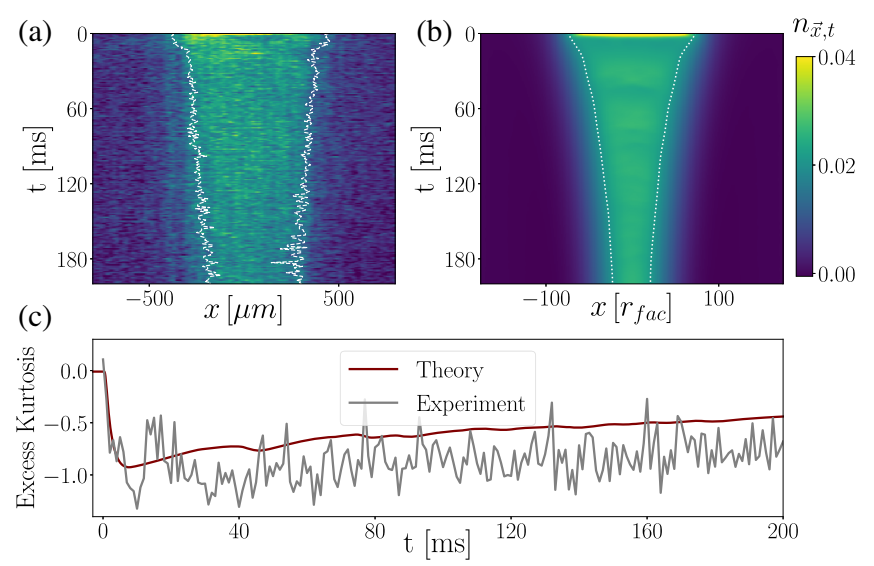

FIG. 3. Melting of the flattop from the time-evolved density profile $n_{\vec{x}, t}$ (projected onto the $x$ axis). (a) Experimental measurements at different times and (b) the simulated evolution show a stable flattop with a lifetime exceeding $200 \mathrm{~ms}$ and a boundary (white dashed curve), which slowly approaches the center. Both plots extend over the same $x$-axis distance. (c) Equilibration of the cloud profile is quantified by its time-dependent excess kurtosis $\mathrm{EK}_{t}=\int d x\left(x / \sigma_{x}\right)^{4} n_{\vec{x}, t}-3$, where we integrate over a density slice with $y=z=0$. Here $\sigma_{x}$ is the width of the cloud in the $x$ direction. Starting from a Gaussian shape $(\mathrm{EK}=0)$, the kurtosis drops to $\mathrm{EK} \approx-1$ after the initial avalanche. It recovers when the cloud evolves back towards an asymptotic thermal, Gaussian state.

theory [60]). This indeed ensures that individual avalanches experience a nearly constant central density over their lifetime. It also ensures that the refilling of the central density happens much faster than off-resonant excitations $(\sim 1 / \tau)$, leading to well-separated avalanches, fulfilling the necessary conditions for SOC [23].

Finally, we analyze the late time dynamics, characterized by a slow melting of the flattop, resulting in the reestablishment of a Gaussian density profile, and the absence of excitation avalanches. We identify the state fulfilling both conditions simultaneously as thermal equilibrium. This occurs when the particle reservoirs represented by the flanks are continuously depleted, leading to a gradual shrinking of the flattop region [Figs. 3(a) and 3(b)]. This can be seen by the evolution of the excess kurtosis EK $_{t}$, shown in Fig. 3(c). A nonzero kurtosis serves as a measure for the deviation of the cloud shape from a thermal Gaussian distribution, i.e., it measures the relative flatness of the distribution. Its relaxation monitors the melting of the flattop towards a robust, thermal equilibrium state without excitation outbursts (corresponding to $\mathrm{EK}_{t}=0$ ). Figure 3(c) shows that the timescale on which a Gaussian distribution is restored exceeds $200 \mathrm{~ms}$. Consequently we infer the lifetime of the SOC state to be at least 10 times longer than the timescale associated with selforganization $(\approx 20 \mathrm{~ms})$.

Conclusion.-We have identified an important additional mechanism which explains how SOC can be sustained in a driven-dissipative ultracold atomic gas by nonequilibrium currents. We show that this generates a flattop density distribution at the SOC critical density, quantitatively confirmed by the hydrodynamic Langevin theory. This demonstrates a novel signature for SOC that could help identify SOC-like behavior in other systems, such as roomtemperature atomic vapors and cold molecular plasmas $[29,62]$. Similar mechanisms may also be at play in very different systems including adaptive neural networks $[30,31]$. The fact that the system naturally evolves to a stable, mostly homogeneous shape combined with the effectiveness of the hydrodynamic Langevin theory will enable more stringent tests of nonequilibrium universality in SOC systems. Alternatively, the interplay between internal and external degrees of freedom could lead to other rich dynamical regimes to test, such as oscillatory behavior associated with SOQC $[26,27,63]$.

K. K. acknowledges support from the National Science Foundation through Grant No. DMR-1723367. T. M. W. acknowledges the French National Research Agency (ANR) through the Programme d'Investissement d'Avenir under Contract No. ANR-17-EURE-0024. This project is part of and supported by DFG SPP 1929 GiRyd through Projects No. DI1745/2-1 and WH141/3-3. S. W. is supported by the "Investissements d'Avenir" programme through the Excellence Initiative of the University of Strasbourg (IdEx) and the University of Strasbourg Institute for Advanced Study (USIAS). M. B. acknowledges support from the Alexander von Humboldt foundation.

[1] H. K. Janssen, Z. Phys. B. 42, 151 (1981).

[2] P. Grassberger, Math. Biosci. 63, 157 (1983).

[3] M. Babadi, E. Demler, and M. Knap, Phys. Rev. X 5, 041005 (2015).

[4] M. C. Tsatsos, P. E. Tavares, A. Cidrim, A. R. Fritsch, M. A. Caracanhas, F. E. A. dos Santos, C. F. Barenghi, and V. S. Bagnato, Phys. Rep. 622, 1 (2016).

[5] H. Kadau, M. Schmitt, M. Wenzel, C. Wink, T. Maier, I. Ferrier-Barbut, and T. Pfau, Nature (London) 530, 194 (2016).

[6] E. Gillman, F. Carollo, and I. Lesanovsky, Phys. Rev. Lett. 125, 100403 (2020).

[7] M. A. Muñoz, R. Dickman, A. Vespignani, and S. Zapperi, Phys. Rev. E 59, 6175 (1999).

[8] K. A. Takeuchi, M. Kuroda, H. Chaté, and M. Sano, Phys. Rev. Lett. 99, 234503 (2007).

[9] G. Lemoult, L. Shi, K. Avila, S. V. Jalikop, M. Avila, and B. Hof, Nat. Phys. 12, 254 (2016).

[10] M. Sano and K. Tamai, Nat. Phys. 12, 249 (2016).

[11] B. Nowak, D. Sexty, and T. Gasenzer, Phys. Rev. B 84, 020506(R) (2011).

[12] P. Bak, C. Tang, and K. Wiesenfeld, Phys. Rev. Lett. 59, 381 (1987).

[13] M. Buchhold and S. Diehl, Phys. Rev. A 92, 013603 (2015). 
[14] J. Berges, A. Rothkopf, and J. Schmidt, Phys. Rev. Lett. 101, 041603 (2008).

[15] O. Kinouchi and M. Copelli, Nat. Phys. 2, 348 (2006).

[16] N. Bertschinger and T. Natschläger, Neural Comput. 16, 1413 (2004).

[17] S. Bornholdt and T. Rohlf, Phys. Rev. Lett. 84, 6114 (2000).

[18] E. Nicklas, M. Karl, M. Höfer, A. Johnson, W. Muessel, H. Strobel, J. Tomkovičfi, T. Gasenzer, and M. K. Oberthaler, Phys. Rev. Lett. 115, 245301 (2015).

[19] P. Bak, C. Tang, and K. Wiesenfeld, Phys. Rev. A 38, 364 (1988).

[20] E. Altshuler and T. H. Johansen, Rev. Mod. Phys. 76, 471 (2004).

[21] S. Field, J. Witt, F. Nori, and X. Ling, Phys. Rev. Lett. 74, 1206 (1995).

[22] S.-K. Jian, S. Yin, and B. Swingle, Phys. Rev. Lett. 123, 170606 (2019).

[23] M. J. Aschwanden, N. B. Crosby, M. Dimitropoulou, M. K. Georgoulis, S. Hergarten, J. McAteer, A. V. Milovanov, S. Mineshige, L. Morales, N. Nishizuka, G. Pruessner, R. Sanchez, A. S. Sharma, A. Strugarek, and V. Uritsky, Space Sci. Rev. 198, 47 (2016).

[24] D. L. Turcotte, Rep. Prog. Phys. 62, 1377 (1999).

[25] G. Pruessner, Self-Organised Criticality: Theory, Models and Characterisation (Cambridge University Press, Cambridge, England, 2012).

[26] J. A. Bonachela and M. A. Muñoz, J. Stat. Mech. (2009) P09009.

[27] J. A. Bonachela, S. de Franciscis, J. J. Torres, and M. A. Muñoz, J. Stat. Mech. (2010) P02015.

[28] S. Helmrich, A. Arias, G. Lochead, T. M. Wintermantel, M. Buchhold, S. Diehl, and S. Whitlock, Nature (London) 577, 481 (2020).

[29] D.-S. Ding, H. Busche, B.-S. Shi, G.-C. Guo, and C. S. Adams, Phys. Rev. X 10, 021023 (2020).

[30] A. Levina, J. M. Herrmann, and T. Geisel, Nat. Phys. 3, 857 (2007).

[31] J. Zierenberg, J. Wilting, and V. Priesemann, Phys. Rev. X 8, 031018 (2018).

[32] H. Schempp, G. Günter, M. Robert-de Saint-Vincent, C. S. Hofmann, D. Breyel, A. Komnik, D. W. Schönleber, M. Gärttner, J. Evers, S. Whitlock, and M. Weidemüller, Phys. Rev. Lett. 112, 013002 (2014).

[33] N. Malossi, M. M. Valado, S. Scotto, P. Huillery, P. Pillet, D. Ciampini, E. Arimondo, and O. Morsch, Phys. Rev. Lett. 113, 023006 (2014).

[34] E. A. Goldschmidt, T. Boulier, R. C. Brown, S. B. Koller, J. T. Young, A. V. Gorshkov, S. L. Rolston, and J. V. Porto, Phys. Rev. Lett. 116, 113001 (2016).

[35] S. Helmrich, A. Arias, and S. Whitlock, Phys. Rev. A 98, 022109 (2018).

[36] The facilitation radius can be approximated as $r_{\text {fac }}=\left(C_{6} / \Delta\right)^{1 / 6}$, where $\Delta$ is the detuning and $C_{6}$ is the van der Waals coefficient.

[37] T. E. Lee, H. Häffner, and M. C. Cross, Phys. Rev. Lett. 108, 023602 (2012).

[38] I. Lesanovsky and J. P. Garrahan, Phys. Rev. Lett. 111, 215305 (2013).

[39] M. Marcuzzi, E. Levi, W. Li, J. P. Garrahan, B. Olmos, and I. Lesanovsky, New J. Phys. 17, 072003 (2015).
[40] M. Marcuzzi, M. Buchhold, S. Diehl, and I. Lesanovsky, Phys. Rev. Lett. 116, 245701 (2016).

[41] R. Löw, H. Weimer, J. Nipper, J. B. Balewski, B. Butscher, H. P. Büchler, and T. Pfau, J. Phys. B 45, 113001 (2012).

[42] R. Heidemann, U. Raitzsch, V. Bendkowsky, B. Butscher, R. Löw, L. Santos, and T. Pfau, Phys. Rev. Lett. 99, 163601 (2007).

[43] A. Urvoy, F. Ripka, I. Lesanovsky, D. Booth, J. P. Shaffer, T. Pfau, and R. Löw, Phys. Rev. Lett. 114, 203002 (2015).

[44] H. Weimer, R. Löw, T. Pfau, and H. P. Büchler, Phys. Rev. Lett. 101, 250601 (2008).

[45] C. Ates, T. Pohl, T. Pattard, and J. M. Rost, Phys. Rev. Lett. 98, 023002 (2007).

[46] T. Amthor, C. Giese, C. S. Hofmann, and M. Weidemüller, Phys. Rev. Lett. 104, 013001 (2010).

[47] M. Gärttner, K. P. Heeg, T. Gasenzer, and J. Evers, Phys. Rev. A 88, 043410 (2013).

[48] C. Simonelli, M. M. Valado, G. Masella, L. Asteria, E. Arimondo, D. Ciampini, and O. Morsch, J. Phys. B 49, 154002 (2016).

[49] R. Faoro, C. Simonelli, M. Archimi, G. Masella, M. M. Valado, E. Arimondo, R. Mannella, D. Ciampini, and O. Morsch, Phys. Rev. A 93, 030701(R) (2016).

[50] I. Lesanovsky and J. P. Garrahan, Phys. Rev. A 90, 011603 (R) (2014).

[51] M. M. Valado, C. Simonelli, M. D. Hoogerland, I. Lesanovsky, J. P. Garrahan, E. Arimondo, D. Ciampini, and O. Morsch, Phys. Rev. A 93, 040701(R) (2016).

[52] G. Pupillo, A. Micheli, M. Boninsegni, I. Lesanovsky, and P. Zoller, Phys. Rev. Lett. 104, 223002 (2010).

[53] T. M. Weber, M. Hning, T. Niederprm, T. Manthey, O. Thomas, V. Guarrera, M. Fleischhauer, G. Barontini, and H. Ott, Nat. Phys. 11, 157 (2015).

[54] M. Buchhold, B. Everest, M. Marcuzzi, I. Lesanovsky, and S. Diehl, Phys. Rev. B 95, 014308 (2017).

[55] See Supplemental Material at http://link.aps.org/ supplemental/10.1103/PhysRevLett.126.123401 for additional details on the numerical integration scheme and the derivation of the coarse-grained equations of motion, which includes Refs. [56,57].

[56] T. Menold, P. Federsel, C. Rogulj, H. Hölscher, J. Fort'agh, and A. Günther, Beilstein J. Nanotechnol. 7, 1543 (2016).

[57] P.-A. Pantel, D. Davesne, S. Chiacchiera, and M. Urban, Phys. Rev. A 86, 023635 (2012).

[58] I. Dornic, H. Chaté, and M. A. Muñoz, Phys. Rev. Lett. 94, 100601 (2005).

[59] K. Klocke and M. Buchhold, Phys. Rev. A 99, 053616 (2019)

[60] For simulations we take a $30 \times 30 \times 600$ spatial grid and a time discretization $\Delta t=0.002$ with $\kappa=5.2, \quad \Gamma=5.3$, $\gamma_{\downarrow 0}=1.5, D=10, \tau=6 \times 10^{-7}, D_{T}=9$.

[61] T. M. Wintermantel, M. Buchhold, S. Shevate, M. Morgado, Y. Wang, G. Lochead, S. Diehl, and S. Whitlock, Nat. Commun. 12, 103 (2021).

[62] R. Wang, M. Aghigh, K. L. Marroqun, K. M. Grant, J. Sous, F. B. V. Martins, J. S. Keller, and E. R. Grant, Phys. Rev. A 102, 063122 (2020).

[63] V. Buendía, S. di Santo, J. A. Bonachela, and M. A. Muñoz, arXiv:2006.03020. 